\title{
Cardiac T2* measurements in patients with iron overload: a comparison of imaging parameters and analysis techniques
}

\author{
Phalla Ou', Yansong Zhao ${ }^{2}$, Sara El Fawal', Puja Banka ${ }^{1}$, Andrew J Powell ${ }^{1 *}$ \\ From 2011 SCMR/Euro CMR Joint Scientific Sessions \\ Nice, France. 3-6 February 2011
}

\section{Introduction}

In patients at risk for iron overload, measurement of myocardial $\mathrm{T}^{*}$ has emerged as an important noninvasive tool to detect preclinical evidence of toxic levels and titrate chelation therapy. Nevertheless, there exists some variation among practitioners in cardiac $\mathrm{T} 2$ * calculation methods.

\section{Purpose}

To examine the impact of different imaging parameters and data analysis techniques on the calculated cardiac R2* $(1 / \mathrm{T} 2 *)$ in patients at risk for cardiac siderosis.

\section{Methods}

The study group consisted of 36 patients with thalassemia syndromes who had undergone clinical MRI assessment of cardiac siderosis using a standardized protocol and who were selected to yield a broad range of cardiac R2* values. Cardiac R2* measurements were performed on a 1.5 Tesla scanner using a ECG-gated, segmented, multiecho gradient echo sequence obtained in a single breath-hold. R2* was calculated from the signal intensity versus echo time data in the ventricular septum on a single mid-ventricular short-axis slice.

\section{Results}

There was excellent agreement between R2* measured with a blood suppression pre-pulse (black blood technique) and without (mean difference $6.0 \pm 10.7 \mathrm{~Hz}$ ). The black blood technique had superior within study reproducibility (R2* mean difference $1.6 \pm 8.6 \mathrm{~Hz}$ versus $2.7 \pm 14.6 \mathrm{~Hz}$ ) and better interobserver agreement $(\mathrm{R} 2 *$ mean difference

\footnotetext{
'Children's Hospital Boston, Boston, MA, USA

Full list of author information is available at the end of the article
}

$3.4 \pm 8.2 \mathrm{~Hz}$ versus $8.3 \pm 16.5 \mathrm{~Hz}$ ). Using the same minimum TE, the use of small $(1.0 \mathrm{~ms})$ versus large $(2.2 \mathrm{~ms})$ echo spacing had minimal impact on cardiac R2* (mean difference $0.3 \pm 8.7 \mathrm{~Hz}$ ). The application of a region of interest versus a pixel-based data analysis had little effect on cardiac R2* calculation (mean difference $8.4 \pm 6.9 \mathrm{~Hz}$ ). With black blood images, fitting the signal curve to a monoexponetial decay or to a monoexponential decay with a constant offset yielded similar R2* values (mean difference 3.4 \pm 8.1 $\mathrm{Hz})$. Figure 1.

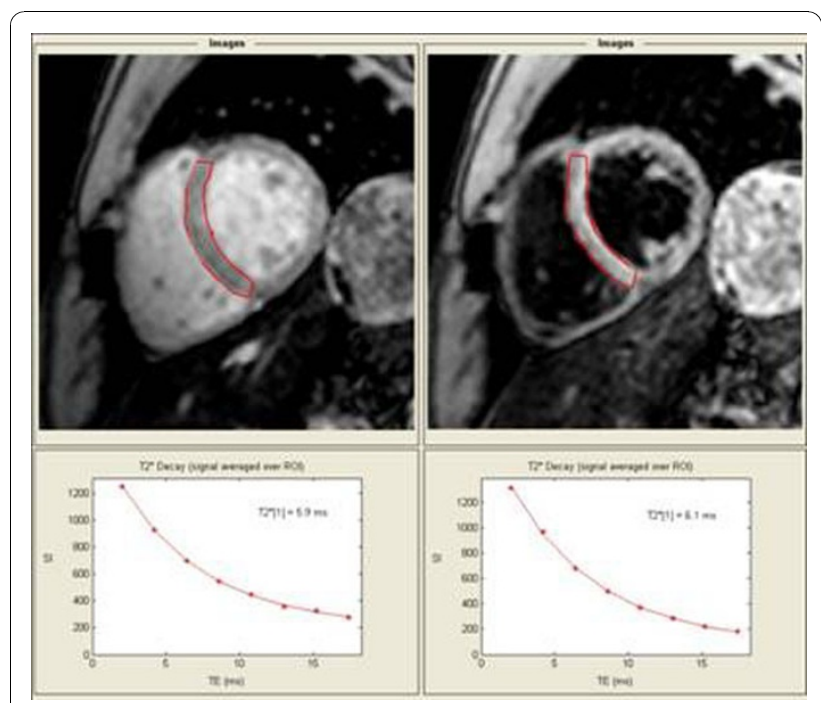

Figure 1 Typical short-axis mid-ventricular cardiac T2* images from the first echo (TE $2.0 \mathrm{~ms}$ ) without and with a blood suppression pre-pulse in the same patient. Below, signal intensity versus TE is plotted for a region of interest encompassing the ventricular septum (outlined in red) along with the decay curve fit to a monoexponential with a constant offset model. 


\section{Conclusions}

The addition of a blood suppression pre-pulse for cardiac R2* measurement yields similar R2* values, and improves reproducibility and interoberver agreement. The findings regarding other variations may be helpful in establishing a broadly accepted imaging and analysis technique for cardiac R2* calculation.

\section{Author details}

${ }^{1}$ Children's Hospital Boston, Boston, MA, USA. ${ }^{2}$ Philips Healthcare, Cleveland, $\mathrm{OH}$, USA.

Published: 2 February 2011

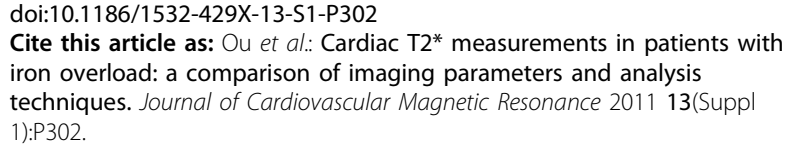

Submit your next manuscript to BioMed Central and take full advantage of:

- Convenient online submission

- Thorough peer review

- No space constraints or color figure charges

- Immediate publication on acceptance

- Inclusion in PubMed, CAS, Scopus and Google Scholar

- Research which is freely available for redistribution

Submit your manuscript at www.biomedcentral.com/submit 